\title{
Targeted Delivery of Adipose Derived Stem Cells into a Transplant by Direct Intra-Arterial Administration
}

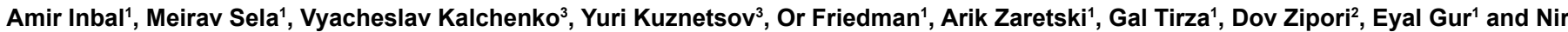 \\ Shani ${ }^{1 *}$
}

${ }^{1}$ The Department of Plastic and Reconstructive Surgery, Tel-Aviv Sourasky Medical Center, affiliated with the Sackler Faculty of Medicine, Tel-Aviv University, Tel-Aviv, Israel

${ }^{2}$ The Department of Molecular Cell Biology, Weizmann Institute of Science, Rehovot, Israel

${ }^{3}$ The Department of Veterinary Resources, In Vivo Optical Imaging Unit, Weizmann Institute of Science, Rehovot, Israel

\begin{abstract}
Objective: Mesenchymal Stem Cells (MSCs) are adult multipotent cells that possess regenerative and immunosuppressant properties. Homing of MSCs to target organs remains a major challenge as intravenous delivery results in intravascular entrapment of most MSCs in vascularized organs. Intra-Arterial (IA) administration of MSCs to arteries feeding a specific organ improved the delivery of cells to these organs but often resulted in vessels obstruction. To improve targeting of MSCs into a transplant we designed a novel method for IA delivery of MSCs during the transplantation procedure. This study was aimed at evaluating the safety and efficacy of this method.

Methods: A syngeneic groin free flap between Lewis rats was performed in all experiment groups. Treatment groups included 3 groups $(n \geq 7)$ in which $1 \times 10^{6}, 0.5 \times 10^{6}$ or $0.05 \times 10^{6}$ adipose derived MSCs (ASCs) were administered via a femoral artery branch prior to the final reperfusion of the flap. In vivo real time fluorescence imaging and intravital microscopy were used to define ASCs IA movement after transplantation.

Results: High concentrations of ASCs per injection resulted in poor flap survival rates $(14.3 \%)$ due to flap necrosis. At $0.05 \times 10^{6} \mathrm{ASC}$, increased long-term flap viability rates $(85 \%)$ were observed. Whole-body imaging of fluorescently labeled ASCs demonstrated significant targeting of cells into the flap even at such a low cell quantity. ASCs were detected in proximity to small blood vessels within the viable flap.
\end{abstract}

Conclusions: Local IA administration of ASCs into a vascularized transplant/flap is feasible and allows high local cell concentrations with minimal cell dosing.

Keywords: Mesenchymal stem cells; Adipose derived mesenchymal stem cells; Vascular composite allotransplantation; Rat groin flap; Intra-arterial

\section{Introduction}

Mesenchymal Stem Cells (MSCs) are multipotent, as evidenced upon culture by their ability to undergo differentiation into adipogenic, osteogenic and chondrogenic fates [1]. The immune modulating properties of MSCs have been repeatedly demonstrated in vitro and in vivo and may be used to prevent allograft rejection by host inflammatory cells and molecules $[2,3]$.

Since the first successful human hand transplantation in 1998 [4], and the first partial human face allotransplantation in 2005 [5], Vascular Composite Allotransplantation (VCA) has gained popularity as a viable alternative for tissue reconstruction. However, VCA transplants are not routinely performed due to the life-long need for immunosuppression with potentially harmful adverse effects such as increased rates of neoplasia and opportunistic infection. Consequently, alternative methods for establishing life-long tolerance while minimizing toxicity are needed. Systemic introduction of mesenchymal stem cells to prevent VCA rejection is a promising alternative evident in in vivo systemic application of adipose derived MSCs (ASCs) to a VCA swine or rat models [3,6-8]. However, despite their proven ability to actively target inflamed and ischemic tissues [9] the vast majority of systemically intravenous (IV) administrated MSCs are found entrapped in vascularized organs such as the lungs and liver with very limited localization of the cells to the target organs [10-13]. Due to this inefficient targeting, MSCs IV administration usually requires large quantities of cells in order to reach therapeutic efficacy, most likely due to the inefficient delivery of cells to the target organs. Thus, developing novel modalities for enhanced targeting of MSCs allowing improved treatments with substantially reduced cell quantities, treatment cost and cell culturing time remains a major challenge in MSCs based cell therapy.

The localization of MSCs to vascularized organs following their IV administration is assumed to be caused largely by their passive entrapment in small vessels due to their large size [9]. An alternative to IV administration is Intra-Arterial (IA) injection to arteries feeding a specific organ. This has shown to increase the quantity of cells that reach the target organ fed by the injected artery [14-18]. Despite the efficient organ targeting by IA administration a large proportion of the cells was still found trapped in the lungs [15]. Additionally, IA administration of MSCs to the brain in animal models of stroke demonstrated increased risk of microvascular occlusion, loss of blood flow and death in association with cell dose [14,16-18].

The aim of the current study was to develop a novel modality that

*Corresponding author: Nir Shani, The Plastic Surgery Department, Tel Aviv Sourasky Medical Center, Tel Aviv 6423906, Israel, Tel: +972 3 6974016; Fax: +972 3 6947504; E-mail: nirs@tlvmc.gov.il

Received November 16, 2016; Accepted November 29, 2016; Published November 30, 2016

Citation: Inbal A, Sela M, Kalchenko V, Kuznetsov Y, Friedman O, et al. (2016) Targeted Delivery of Adipose Derived Stem Cells into a Transplant by Direct Intra-Arterial Administration. J Stem Cell Res Ther 6: 367. doi: 10.4172/2157 7633.1000367

Copyright: (c) 2016 Inbal A, et al. This is an open-access article distributed under the terms of the Creative Commons Attribution License, which permits unrestricted use, distribution, and reproduction in any medium, provided the original author and source are credited. 
will allow enhanced targeting of MSCs into a transplanted allogeneic tissue/organ or alternatively into an autologous free flap. This was achieved by harnessing the tendency of MSCs to entrap in small vessels due to their large size and was based on IA delivery of cells to the transplanted free flap immediately following anastomosis and prior to flap reperfusion. This improved IA delivery system may be adapted to any vascularized flap on the one hand or any tissue or organ transplant on the other.

\section{Materials and Methods}

\section{Animals}

Fifty inbred Lewis rats (LEW; RT1l strain) aged 12 weeks and weighing 280-320 g were used. The study was conducted in accordance with the Guide for the Care and Use of Laboratory Animals and approved by our Institutional Animal Care and Use Committee protocol in Tel Aviv Sourasky Medical Center or Weizmann Institute of Science.

\section{Animal flap model}

The well-described groin free flap rat model was used [19]. Syngeneic groin free flaps were performed between a Lewis donor and a recipient Lewis rat. A groin free flap based on the femoral artery and vein was harvested from the donor rat. This composite flap was raised over the abdominal muscular fascia and included skin, panniculus carnosum and subcutaneous fat (Figure 1). Immediately after donor flap harvest and flap femoral vessels division, irrigation with $10 \mathrm{ml}$ Ringer Lactate; $50 \mathrm{U}$ heparin was performed via the flap's femoral artery until clear fluid could be seen coming out of the femoral vein. In the recipient rat, the proximal femoral vein and artery including its 1 $\mathrm{cm}$ branch were dissected and prepared for anastomosis. The recipient's "arterial branch" (An arterial branch off the femoral artery proximal to the superficial inferior epigastric artery branch) was temporarily closed distally with a 10-0 nylon suture for later local arterial injection of ASCs. After donor flap transfer to the recipient rat, the donor vessels were anastomosed in an end-to-end fashion onto the host femoral vessels with a 10-0 nylon interrupted suture (Figure 2A). At this stage the ASCs were administered into the flap as described below. Following ASCs administration, the flap was inset using 4-0 interrupted suture.

\section{ASCs injection model}

Injection into the flap was performed immediately after vascular anastomosis and a positive milking flow test. Arterial inflow to the flap was arrested by placing a vascular clamp proximal to the anastomosis and the arterial branch. Next, the distally placed suture on the arterial branch was taken off, leaving its end patent for local arterial injection. A blunt 30-gauge needle was introduced into the arterial branch, located proximal to the anastomosis and distal to the arterial vascular clamp (Figure 2B). In order to achieve a steady and accurate application of the cells, the injection was performed by the microsurgeon stabilizing the needle in the arterial branch lumen as his assistant pushed on the syringe gently till its content was emptied (Figure 2C). Next, the patent arterial branch was closed with a nylon 10-0 suture adjacent to its origin from the femoral artery. After 10 minutes of arrested flow, allowing the cells to settle in, the arterial vascular clamp was released permitting reperfusion to occur. Subsequently the skin paddle was sutured into place as described above.

\section{Culturing of ASCs}

As previously described [20], Adipose-derived Stem Cells (ASCs) were extracted from subcutaneous fat tissue of Lewis rats using $0.1 \%$ collagenase (Sigma, St. Louis, MO, USA). ASCs were cultured in high glucose Dulbecco's modified Eagle's medium (DMEM; Gibco, Paisley, Scotland, UK) supplemented with $10 \%$ FCS (Thermo Scientific HyClone, Tauranaga, New Zealand). Cultures were propagated at $37^{\circ} \mathrm{C}$ in a $10 \% \mathrm{CO} 2$ atmosphere.

\section{ASCs differentiation}

Adipogenic differentiation: Confluent cells were cultured in adipogenic medium containing $10 \mu \mathrm{g} / \mathrm{mL}$ insulin, $1 \times 10^{-6} \mathrm{M}$ dexamethasone, $0.5 \mathrm{mM}$ IBMX (3-isobutyl-1-methyl xanthine) and $50 \mu \mathrm{M}$ indomethacin (all from Sigma). After 21 days, the cells were fixed by $4 \%$ formalin (20 $\mathrm{min}$ at room temperature [RT]) and stained with $0.5 \%$ Oil Red (10 min, at RT) (Sigma). Following the staining cells

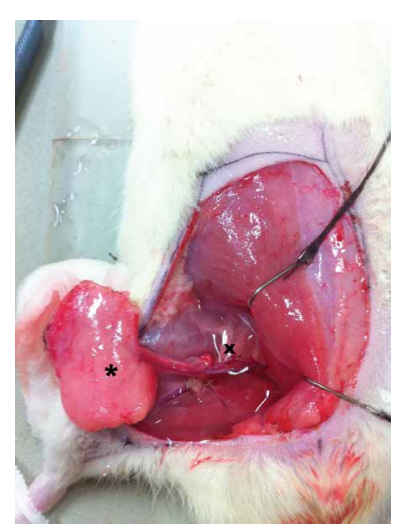

Figure 1: Groin free flap harvest based on the femoral vessels $(\mathbf{x})$. This composite flap $\left(^{*}\right)$ is raised over the abdominal muscular fascia includes skin, panniculus cornosum and subcutaneous fat.
A.

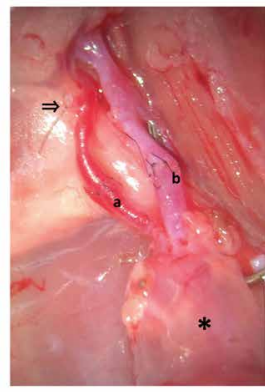

C.

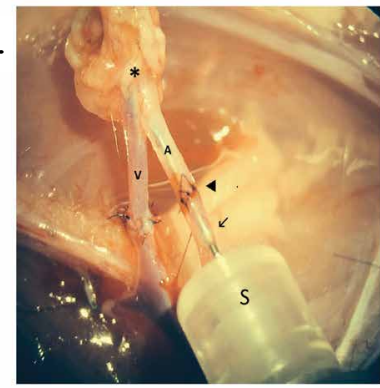

B.

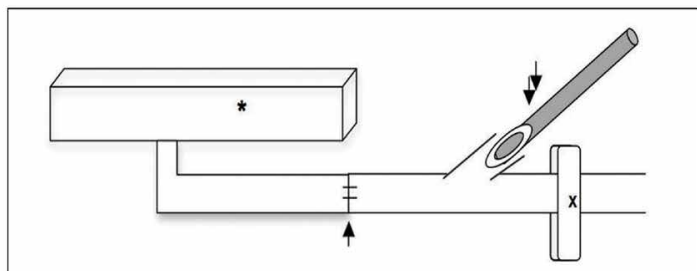

Figure 2: (A) Recipient arterial branch $(\Rightarrow)$ temporarily closed distally for later local arterial injection of ASCs. The donor flap (*) femoral artery (a) and vein (b) were anastomosed end-to-end to recipient vessels under microscopic magnification. (B) Schematic representation, following flap $\left(^{*}\right)$ anastomosis, of the 30-gauge blunt needle $(\leftarrow)$ introduced into the arterial branch, proximal to the anastomosis $(\leftarrow)$ and distal to the arterial vascular clamp $(\mathbf{x})$. (C) In order to achieve a steady and accurate application of the cells, the injection was performed by the microsurgeon stabilizing the needle in the arterial branch $(\boldsymbol{K})$ lumen as his assistant pushes on the syringe $(\mathbf{S})$ gently till its content is emptied. Anastomosis (४); Flap artery (A); Flap vein (V); Flap pedicle (*). 
were photographed (Olympus IX71 microscope with a DP73 camera) and Oil red O was extracted by 4\% IGEPAL (Sigma) in Isopropanol and quantified at $520 \mathrm{~nm}$ using a TECAN Infinite M200 plate reader (TECAN, Männedorf, Switzerland).

Osteogenic differentiation: Confluent cells were cultured in StemPro ${ }^{\oplus}$ Osteogenesis Differentiation Kit (Gibco). After 21 days, the cells were fixed with $4 \%$ formalin $(20$ min at RT) and stained with $2 \%$ Alizarin Red (Sigma), pH 4.2 (10 min at RT). The induction medium was replaced every 3-4 days in all cases. Photographs were taken using an Olympus IX71 microscope with a DP73 camera.

Flow cytometry. Surface marker analysis: Cells were harvested and incubated with a 4-color panel containing CD90.1-pacific blue and CD29-APC (Miltenyi Biotec, Auburn, CA, USA), CD45-APC/ cy7 (Biolegend, San Diego, CA, USA) and live/dead-aqua blue dye (Invitrogen molecular probes, Eugene, OR, USA) for 30 min. Data analysis was performed by the flow-Jo software (Tree star, Ashland, OR USA).

\section{Experiment design}

All rats underwent transplantation with a syngeneic groin free flap. In the control group, (group I; $\mathrm{n}=4$ ), $200 \mu \mathrm{l}$ of saline was administered intra-arterially through the arterial branch immediately following the vascular anastomosis.

In the treatment groups ASC were administered intra-arterially through the arterial branch immediately following vascular anastomosis. ASCs were administered in $200 \mu \mathrm{l}$ saline in the following groups: Group II $1 \times 10^{6}$ ASCs $(n=7)$, Group III $0.5 \times 10^{6}$ ASCs $(n=8)$ and group IV $0.05 \times 10^{6} \mathrm{ASCs}(\mathrm{n}=8)$.

\section{Flap viability}

The transplanted groin flap was observed daily for signs of flap ischemia and necrosis as defined by the well-characterized sequence of swelling, discoloration, epidermolysis, dyskeratosis, and necrosis. A flap that survived 10 days post-harvest was considered to be permanently viable (Figure 3I). Flap failure due to complete vessel occlusion was apparent by flap necrosis at post-transplant day 2-3 (Figure 3II) demonstrating an early, clinically visible, ischemic assault.

\section{Imaging studies}

Whole body near infrared imaging: ASCs were labeled by a near Infrared (IR) fluorescent, lipophilic carbocyanine $\operatorname{DiOC18}_{(7)}$ (DIR) dye (Molecular Probes) Life Technologies, Carlsbad, CA, USA, which is weakly fluorescent in water but highly fluorescent when incorporated into lipoid cell membranes, was used according to [21,22] DIR ]has absorption and fluorescence maxima at 750 and $782 \mathrm{~nm}$, respectively, which corresponds to low light absorption and auto-fluorescence in living tissues. Labeled cells were intra-arterially administered into the flap and imaging was performed using an in vivo pre-clinical imaging system Xenogen's IVIS SPECTRUM 100 Series Imaging System (Caliper Life Sciences, Alameda, California, currently Perkin Elmer) immediately after flap anastomosis and 24 hours post-transplantation. Viability of all cells was examined following their labeling and prior to their administration by Trypan blue staining (Sigma-Aldrich, Israel).

Intravital microscopy: ASCs were labeled by Carboxyfluorescein Succinimidyl Ester (CFSE) (Invitrogen, CA, USA) and their viability was examined following their labeling and prior to their administration by Trypan blue staining (Sigma-Aldrich, Israel). CFSE labeled cells were intra-arterially administered into the flap and Combined Fluorescent
Intravital Microscopy (FIM) and Laser Speckle Imaging (LSI) were performed on a modified zoom stereomicroscope SZX12 (Olympus, Japan) coupled with the CCD camera PIXELFLY QE, 12 bit (PCO, Germany) to track the labeled ASCs 23-27. A FIM mode, excitation/ emission filter set was adjusted as 460-490/ 510-550 nm, respectively. LSI is an advanced analog of Laser Doppler Imaging and it was used for obtaining additional information about blood perfusion in each flap. Image acquisition and processing during LSI mode were performed as previously described in the literature [23-27].

Fluorescent vascular studies: To better define the flap perfusion, fluorescein sodium salt (Sigma-Aldrich, Israel) was administrated in a bolus injection through the rat's tail vein $(2 \mathrm{mg} / \mathrm{kg}$ in a volume of $0.5 \mathrm{ml})$.

\section{Statistical analysis}

Fisher's exact test was used to assess the statistically significant differences between experimental groups. A P value of $<0.05$ was considered to be statistically significant.

\section{Results}

Fate of groin free flap following direct arterially injected ASCs: To prove the feasibility and safety of our novel IA modality, $200 \mu \mathrm{l}$ of saline only was administered through the arterial branch immediately following vascular anastomosis in the control group (group I; $n=4$ ). All flaps survived and were viable at postoperative day 10 .
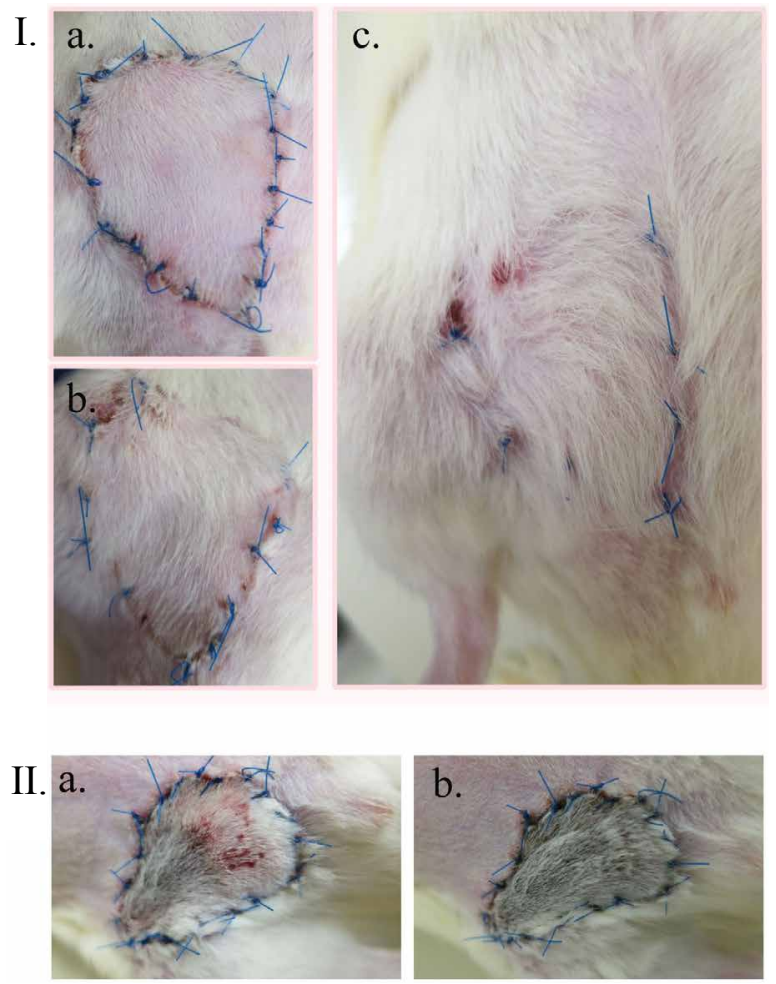

Figure 3: Clinical evaluation of syngeneic flap survival following ASC administration determines the level of vessel occlusion by administered cells. (I) A syngeneic flap that survived 10 days post-harvest was considered to be permanently viable. (a) post-operative day 2; (b) post-operative day 6; (c) post-operative day 13. (II) Flap failure due to vessel occlusion was recorded at post-transplant day 2-3 demonstrating swelling and discoloration in postoperative day 2 (a) and epidermolysis, dyskeratosis, and necrosis in postoperative day $3(\mathbf{b})$. 
In the treatment groups each rat received a volume of $200 \mu \mathrm{l}$ saline with decreasing concentration of ASCs (ASCs characterization is shown in supplementary Figure 1). As can be seen in Figure 4 and in Supplementary Table 1, administration of $1 \times 10^{6}$ cells (group II; $n=7)$ resulted in a low flap survival rate $(14.3 \%)$ due to vessel occlusion followed by flap necrosis and death. Reducing the quantity of administered cells to $0.5 \times 10^{6}$ ASCs resulted in increased flap survival, although flap death was still observed in $50 \%$ of the animals (group III; $\mathrm{n}=8$ ). Flap survival rates further increased to $75 \%$ in flaps injected with a lower cell quantity of $0.05 \times 10^{6}$ (group IV), demonstrating a significant $(\mathrm{p}=0.045)$ correlation between the amount of administered cells and the rate of flap survival.

\section{Fate of Labeled Injected ASCs}

Macroscopic whole-body imaging with DIR labeled ASCs at $1 \times$ $10^{6}$ administered dose $(\mathrm{n}=2)$ were studied to better understand the in vivo fate of ASCs. ASCs remained concentrated in the flap and could not be observed in any other sites, such as the lung or liver, when imaged immediately after injection and again 24 hours later (Figure 5). However, a vascular flow study of the transferred flap revealed that although perfusion was intact immediately after injecting the ASCs, it was completely impeded on the arterial side after 24 hours, indicating a subclinical ischemic event. Dissection of the flap's vasculature revealed a clotted artery and vein with no perfusion. At a concentration of 0.05 $\times 10^{6}$ whole-body imaging with DIR labeled ASCs $(n=3)$ again showed cells to be concentrated within the flap immediately after injection and 24 hours later (Figure 6Ia and 6Ic respectively). To confirm that the vascular flow was not disturbed at this cell concentration, a green fluorescent dye was systemically administered IV and its appearance in the flap skin was examined. Fluorescence within the flap skin was of similar intensity to the surrounding normal skin revealing intact perfusion 20 minutes and 24 hours after injecting the ASCs (Figure 6Ib and 6Id respectively).

To better define ASCs location within the transplanted flap, cells were labeled with CFSE, a membrane permeable green fluorescent dye.

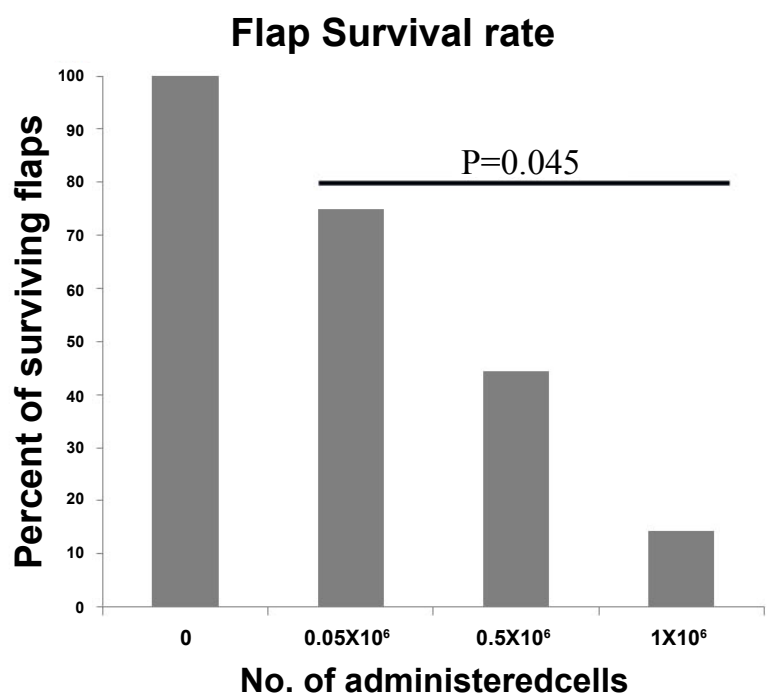

Figure 4: Flap survival is affected by the number of ASCs administered. Syngeneic flap transplantation was performed and flaps were injected with either saline only (group I) or $1 \times 10^{6} \mathrm{ASCs}$ (Group II), $0.5 \times 10^{6}$ (group III) or $0.05 \times 10^{6}$ (group IV). The percent of surviving flaps in each group is presented. $P$ value was calculated using Fisher's exact test.
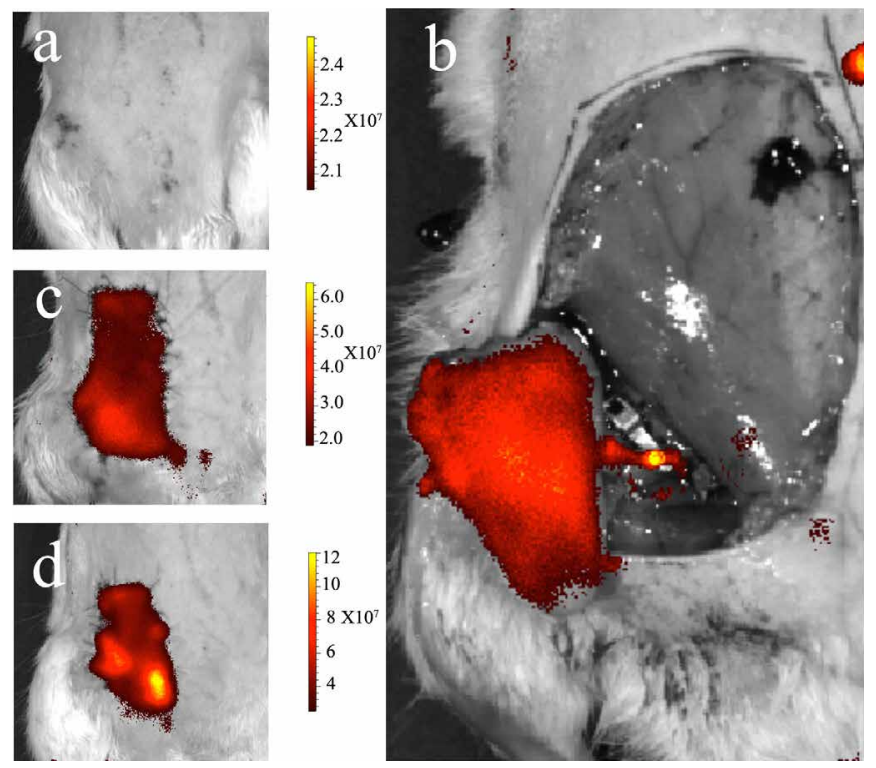

Figure 5: IA administered ASCs $\left(1 \times 10^{6}\right)$ were detected within the flap but eventually led to blood flow occlusion. IA administration of DIR labeled ASCs was performed during a syngeneic groin flap transplantation. Fluorescent signal was detected by whole body in vivo imaging immediately following surgery (b), at 20 minutes (c) and at 24 hours (d) after surgery. Whole body image before injection served as a background control (a).

CFSE labeled ASCs were followed by intravital microscopy revealing ASCs localization in the vicinity of vessels at the capillary level (Figure 6II). Imaging of CFSE labeled cells in vivo demonstrated also the viability of the ASCs following their administration. Direct microscopic Doppler flow study demonstrated intact flap perfusion 24 hours after surgery and ASCs administration (Figure 7). At postoperative day 10 these three imaged flaps injected with a concentration of $0.05 \times 10^{6}$ cells were viable and intact.

\section{Discussion}

MSCs have been shown to serve as regenerative and immunosuppressive tools both in vitro and in vivo [28]. MSCs application for immunosuppressive purposes in both clinical practice in humans and animal research include mainly systemic delivery via a peripheral vein leading to the entrapment of most injected MSCs in the lung's pre capillaries at their first pass [10-13]. These MSCs reach their destination in decreased concentration and lowered bioavailability at the target tissue [12]. Previous studies demonstrated improved organ targeting of MSCs by IA administration into an intact artery feeding an organ [14-18]. However, large quantities of cells were still required to achieve significant organ detainment by IA administration and the majority of cells were still reported to reach the lungs [15]. To enhance MSCs entrapment within a vascularized groin flap we thus utilized the inherent characteristics of the transplantation procedure and delayed flap reperfusion following IA cell administration. This allowed the cells to settle within the blood vessels prior to the blood flow reconstitution. Although seemingly easier, we did not attempt to administer the cells directly into the pedicle artery prior to anastomosis, due to the long and unstandardized anastomosis time that was required following cell administration. Using our technique we were able to demonstrate strong targeting of ASCs to the transplanted flap. Importantly, however, administration of high quantities of cells to the transplant led to vessel occlusion and flap necrosis. Using reduced cell quantities we were able 
Citation: Inbal A, Sela M, Kalchenko V, Kuznetsov Y, Friedman O, et al. (2016) Targeted Delivery of Adipose Derived Stem Cells into a Transplant by Direct Intra-Arterial Administration. J Stem Cell Res Ther 6: 367. doi: 10.4172/2157-7633.1000367

Page 5 of 7

I. a.
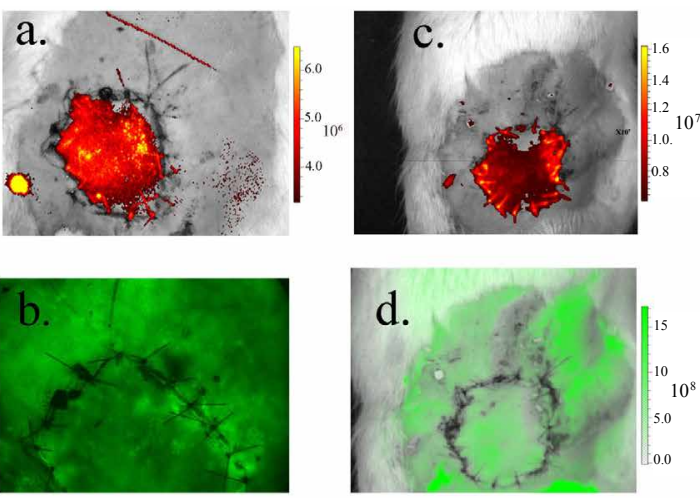

II.

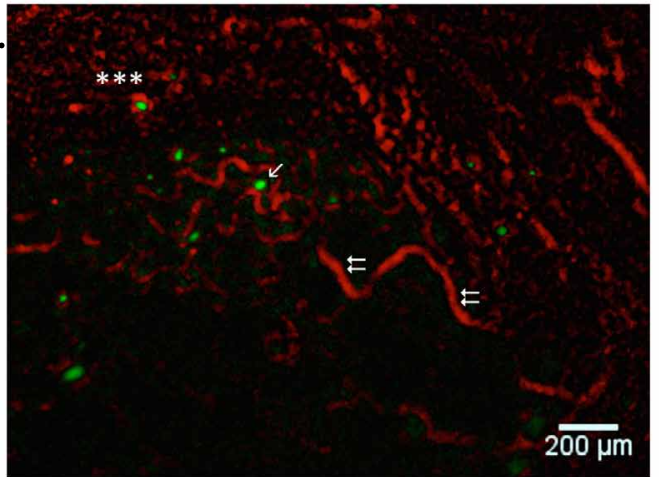

Figure 6: (I) ASCs remained in the viable flap following administration of 0.05 $\times 10^{6}$ DIR labeled ASCs and did not disturb blood flow to the flap as seen 20 minutes (a) after injection as well as 24 hours (c) later; Image taken by IVIS. Green fluorescent dye injected systemically, revealed that the flap's perfusion was intact after 20 minutes (b) as well as after 24 hours (d); images were taken by fluorescence intravital microscopy after the injection of cells (b) and IVIS (d). (II) Intravital microscopy of CFSE labeled ASCs (green labeled cells, $\boldsymbol{K}$ ) presented intact perfusion (red labeled vessels, $\leftarrow$ ) with well-defined visualized ASCs localized in vicinity of the capillaries. Flap subcutaneous tissue marked $\left.{ }^{* * *}\right)$.

to achieve good cell targeting to the flap without critically affecting its blood perfusion and achieving high rates of flap survival. Thus, our novel IA administration technique provides a feasible alternative to IV administration achieving efficient and safe targeting into a transplant with limited cell quantities.

Prior to the beginning of our study and during the construction of our novel delivery method, injecting the cells through the arterial pedicle was attempted, with the goal to ease the technique. The shortcoming with injecting the cells into the arterial pedicle prior to performing the anastomosis was that, this resulted in long and uncontrolled duration of blood flow cessation due to the different anastomotic challenges. We decided that the most controlled manner for cells injection was after completion of the anastomosis and following a satisfying patency-flow test. Different methods for cells injection following the anastomosis were tried and resulted in inferior injection ease. Puncturing the proximal recipient femoral artery led to bleedings and vessel injuries which we thought could lead to results bias. Furthermore, removing some anastomotic sutures also led to more injury to the already divided vessel edge. It seemed to us that the least traumatic approach was to cannulate the arterial branch leading to the pedicle. After injection, the branch was divided at its origin. This served us in a very consistent way in relation to flow cessation duration and a-traumatic cell delivery.

We anticipated that the major mechanism influencing cell detainment within the flap vasculature following IA administration would be passive entrapment. However, the delayed reperfusion allows also time for the administered cells to settle and adhere to blood vessels within the flap also by active arrest mechanisms through various surface proteins [9]. We speculated that delaying blood reperfusion to the flap after IA cell administration and letting them settle within the flap's vasculature would allow their enhanced retention once blood flow is reconstituted. Indeed using this protocol we were able to observe strong targeting of the cells to the flap. Macroscopic whole-rat imaging with DIR labeled ASCs (group III) revealed that the cells were concentrated within the flap immediately following injection and also 24 hours later in a viable and well-perfused flap. Importantly a strong florescent signal was observed even when as little as $0.05 \times 10^{6}$ cells were injected into the flap indicating strong cell retention. Thus, despite a 20 -fold reduction in ASCs administered compared to systemic delivery methods, significant intra-flap ASCs delivery was still achieved.

Previous reports demonstrated that the massive MSC entrapment in the lungs following their IV administration was reduced dramatically 24 hours post transplantation (around 10\% of the signal remained) $[11,29]$. An apparent reduction of the cell signal within the flap was observed also in the current study between time 0 and 24 hours post their administration (Figure 6Ia and 6Ic). As was summarized in a recent review [30] MSCs survival in vivo is limited and most of the cells disappear rapidly. Importantly, despite the rapid clearance of most cells, a strong and repetitive immunosuppressive effect of MSCs is still evident in both animal models and in the clinic [31,32]. Thus, we assume that the significant targeting of ASCs to the flap, as was evident immediately following their administration and at 24 hours, would suffice for an improved immunosuppressive effect compared to IV/ systemic administration that achieves only limited specific localization of cells to target organ/tissue.

IA administration of cells to ischemic brain led to blood flow

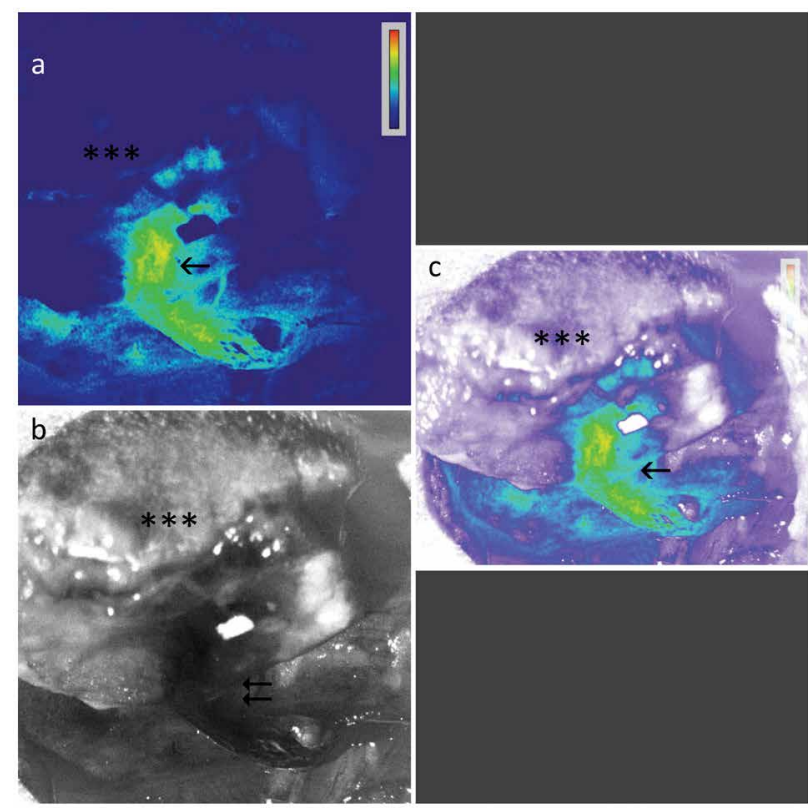

Figure 7: Doppler flow study 24 hours after surgery and administration of 0.05 $\times 10^{6}$ ASCs demonstrated intact flap perfusion. (I) Doppler study presenting intact inflow and outflow $(\leftarrow)$ from the flap $\left.{ }^{(* *}\right)$. (II) Raw image of flap $\left(^{* * *}\right)$

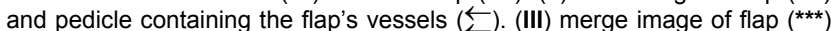
and pedicle $(\leftarrow)$. 
Citation: Inbal A, Sela M, Kalchenko V, Kuznetsov Y, Friedman O, et al. (2016) Targeted Delivery of Adipose Derived Stem Cells into a Transplant by Direct Intra-Arterial Administration. J Stem Cell Res Ther 6: 367. doi: 10.4172/2157-7633.1000367

Page 6 of 7

obstruction especially when large quantities of cells were entrapped within the brain [14,16-18].

Due to the high rates of cell entrapment in our model we anticipated an increased risk of blood flow obstruction. As a syngeneic rather than an allogeneic flap model was used, all necrosed flaps were diagnosed as an ischemic failure. Indeed, we found high flap failure rates at high administered MSCs quantities while flap viability increased dramatically when a lower MSCs quantity was administered. Similarly to our findings, a recent report suggested that only cell doses lower than $0.1 \times 10^{6}$ do not completely compromise blood flow and can be considered safe [18]. The direct correlation between the administered cell dose and the blood flow reduction was further demonstrated in other studies $[14,16,17]$. Thus our proposed model allows the delivery of a high proportion of injected cells directly into a viable transplant/ flap using very low cell quantities.

As was appreciated in recent years, MSCs produce and secrete innumerable growth factors and cytokines. This paracrine activity of MSCs is suggested to be responsible for many of their regenerative as well as their immunosuppressive properties [33]. The proximity of MSCs to the target tissue is therefore of great importance in order to allow an effective paracrine effect. The potential use of MSCs for the prevention of tissue or solid organ allograft rejection has already been shown in animal models [7,8,34-38].

Recent study has shown that inhibition of the catalytic activity of histone methyltransferase G9a promotes the differentiation of adipose-derived mesenchymal stem cells towards endothelial cells [39]. Moreover, as compared with normal human ASCs, blocking the function of G9a also leads to the up-regulation of factors involved in blood vessel formation [39], it would therefore be of importance to investigate in the future whether ASCs pre-treated with G9a inhibitor prior to their transplantation could increase the efficiency and successfulness of syngeneic groin free flap transplantation.

VCA such as hand or face transplantation is an elective procedure performed with the goal of improving patient's quality of life, in contrast to solid organ transplants which are performed in order to prolong the patient's lifespan. Utilization of the immunosuppressive qualities of MSCs may offer a novel nontoxic strategy to avoid the lifelong need for immunosuppressant drugs or to decrease daily dosage in order to consequently minimize toxicity in VCA. We speculate that our novel IA delivery technique could potentially enhance MSC ability to prevent allotransplant rejection, due to its ability to increase their targeting to the proximity of the rejection site i.e. the donor recipient interface.

\section{Conclusions}

Our data indicates that IA ASCs local delivery at a correct and calibrated concentration can serve as a therapeutic option for better stem cell delivery into a transplant or flap. The substantial increase in the rate of flap survival in correlation with reduced cell administering suggests also that the proposed method is feasible with limited risks. However, it clearly demonstrates the possible risk of vessel obstruction when high cells quantities are administrated. Therefore prior to its application in humans, the method must be adjusted in a more compatible large animal model.

\section{Financial Disclosure Statement}

None of the authors has a financial interest in any of the products, devices, or drugs mentioned in this manuscript.

\section{Author Contribution}

Al designed the local ASCs administration experiments and performed them together with $A Z$ and OF. EG together with $A I$ and NS conceived the intravascular delivery method and drafted the manuscript. MS and GT prepared, characterized and labeled rat ASCs for the local administration experiments. VK and $Y K$ performed the in vivo and intravital imaging and analysis. NS, DZ and EG designed and coordinated the study. All authors critically read and approved the final manuscript.

\section{References}

1. Pittenger MF, Mackay AM, Beck SC, Jaiswal RK, Douglas R, et al. (1999) Multilineage potential of adult human mesenchymal stem cells. Science 284 143-147. [PubMed]

2. François M, Galipeau J (2012) New insights on translational development of mesenchymal stromal cells for suppressor therapy. J Cell Physiol 227: 35353538. [PubMed]

3. Kuo YR, Chen CC, Shih HS, Goto S, Huang CW, et al. (2011) Prolongation of composite tissue allotransplant survival by treatment with bone marrow mesenchymal stem cells is correlated with t-cell regulation in a swine hind-limb model. Plast Reconstr Surg 127: 569-579. [PubMed]

4. Dubernard JM, Owen E, Herzberg G, Lanzetta M, Martin X, et al. (1999) Human hand allograft: Report on first 6 months. The Lancet 353: 1315-13 20. [PubMed]

5. Dubernard JM, Lengelé B, Morelon E, Sylvie Testelin, Lionel Badet, et al (2007) Outcomes 18 months after the first human partial face transplantation. N Engl J Med 357: 2451-2460.

6. Cheng HY, Ghetu N, Huang WC, Wang YL, Wallace CG, et al. (2014) Syngeneic adipose-derived stem cells with short-term immunosuppression induce vascularized composite allotransplantation tolerance in rats. Cytotherapy 16: 369-380. [PubMed]

7. Kuo YR, Chen CC, Goto S, Lee IT, Huang CW, et al. (2011) Modulation of immune response and $t$-cell regulation by donor adipose-derived stem cells in a rodent hind-limb allotransplant model. Plast Reconstr Surg 128: 661e-672e. [PubMed]

8. Kuo YR, Goto S, Shih HS, Wang FS, Lin CC, et al. (2009) Mesenchymal stem cells prolong composite tissue allotransplant survival in a swine model. Transplantation 87: 1769-1777. [PubMed]

9. Karp JM, Teo GSL (2009) Mesenchymal stem cell homing: The devil is in the details. Cell stem cell 4: 206-216. [PubMed]

10. Barbash IM, Chouraqui P, Baron J, Feinberg MS, Etzion S, et al. (2003) Systemic delivery of bone marrow-derived mesenchymal stem cells to the infarcted myocardium feasibility, cell migration, and body distribution. Circulation 108: 863-868. [PubMed]

11. Kraitchman DL, Tatsumi M, Gilson WD (2005) Dynamic imaging of allogeneic mesenchymal stem cells trafficking to myocardial infarction. Circulation 112 1451-1461. [PubMed]

12. Lee RH, Pulin AA, Seo MJ, Ishimori T, Kedziorek D, et al. (2009) Intravenous hmscs improve myocardial infarction in mice because cells embolized in lung are activated to secrete the anti-inflammatory protein tsg-6. Cell stem cell 5 : 54-63. [PubMed]

13. Raymond JGJED, Lundberga FMM, Caplana Al (2001) The dynamic in vivo distribution of bone marrow-derived mesenchymal stem cells after infusion. Cells Tissues Organs 169: 12-20. [PubMed]

14. Cui L, Kerkelä E, Bakreen A, Nitzsche F, Andrzejewska A, et al. (2015) The cerebral embolism evoked by intra-arterial delivery of allogeneic bone marrow mesenchymal stem cells in rats is related to cell dose and infusion velocity. Stem Cell Research \& Therapy 6: 11

15. Hara M, Murakami T, Kobayashi E (2008) In vivo bioimaging using photogenic rats: Fate of injected bone marrow-derived mesenchymal stromal cells. J Autoimmun 30: 163-171. [PubMed]

16. Li L, Jiang Q, Ding G, Zhang L, Zhang ZG, et al. (2010) Effects of administration route on migration and distribution of neural progenitor cells transplanted into rats with focal cerebral ischemia, an mri study. J Cereb Blood Flow Metab 30: 653-662. [PubMed]

17. Walczak P, Zhang J, Gilad AA, Kedziorek DA, Ruiz-Cabello J, et al. (2008) Dual-modality monitoring of targeted intraarterial delivery of mesenchymal stem cells after transient ischemia. Stroke 39:1569-1574. [PubMed] 
Citation: Inbal A, Sela M, Kalchenko V, Kuznetsov Y, Friedman O, et al. (2016) Targeted Delivery of Adipose Derived Stem Cells into a Transplant by Direct Intra-Arterial Administration. J Stem Cell Res Ther 6: 367. doi: 10.4172/2157-7633.1000367

18. Yavagal DR, Lin B, Raval AP, Garza PS, Dong C, et al. (2014) Efficacy and dose-dependent safety of intra-arterial delivery of mesenchymal stem cells in a rodent stroke model. PloS one 9: e93735. [PubMed]

19. Zhang F, Sones WD, Lineaweaver WC (2001) Microsurgical flap models in the rat. J Reconstr Microsurg 17: 211-222. [PubMed]

20. Sela M, Tirza G, Ravid O, Volovitz I, Solodeev I, et al. (2015) Nox1-induced accumulation of reactive oxygen species in abdominal fat derived mesenchymal stromal cells impinges on long-term proliferation. Cell Death Dis 6: e1728. [PubMed]

21. Kalchenko V, Neeman M, Harmelin A (2011) Whole-body imaging of hematopoietic and cancer cells using near-infrared probes. Advanced fluorescence reporters in chemistry and biology iii, Springer pp: 329-346.

22. Kalchenko V, Shivtiel S, Malina V, Lapid K, Haramati S, et al. (2006) Use of lipophilic near-infrared dye in whole-body optical imaging of hematopoietic cell homing. J Biomed Opt 11: 050507.

23. Kalchenko V, Israeli D, Kuznetsov Y, Harmelin A (2014) Transcranial optical vascular imaging (tovi) of cortical hemodynamics in mouse brain. Scientific reports

24. Kalchenko V, Madar-Balakirski N, Meglinski I, Harmelin A (2011) In vivo characterization of tumor and tumor vascular network using multi-modal imaging approach. J Biophotonics 4: 645-649. [PubMed]

25. Kalchenko V, Ziv K, Addadi Y, Balakirsiki NM, Melginski I, et al. (2010) Combined application of dynamic light scattering imaging and fluorescence intravital microscopy in vascular biology. Laser Physics Letters 7: 603.

26. Milo I, Sapoznikov A, Kalchenko V (2013) Dynamic imaging reveals promiscuous crosspresentation of blood-borne antigens to naive cd8+t cells in the bone marrow. Blood 122:193-208. [PubMed]

27. Tew GA, Klonizakis M, Crank H, Briers JD, Hodges GJ (2012) Comparison of laser speckle contrast imaging with laser doppler for assessing microvascular function. Microvasc Res 82: 326-332.

28. Wei X, Yang X, Han Z-p, Qu FF, Shao L, et al. (2013) Mesenchymal stem cells: A new trend for cell therapy. Acta Pharmacologica Sinica 34: 747-754. [PubMed]
29. Eggenhofer E, Benseler V, Kroemer A, Popp FC, Geissler EK, et al. (2012) Mesenchymal stem cells are short-lived and do not migrate beyond the lungs after intravenous infusion. Frontiers in immunology 3: 297 [PubMed]

30. Eggenhofer E, Luk F, Dahlke MH, Hoogduijn MJ (2014) The life and fate of mesenchymal stem cells. Frontiers in immunology 5: 148 [PubMed]

31. Ghannam S, Bouffi C, Djouad F, Jorgensen C, Noël D (2010) Immunosuppression by mesenchymal stem cells: Mechanisms and clinical applications. Stem Cell Res Ther 1: 2. [PubMed]

32. Ren G, Chen X, Dong F, Li W, Ren X, et al. (2012) Concise review: Mesenchymal stem cells and translational medicine: Emerging issues. Stem Cells Transl Med 1: 51-58. [PubMed]

33. Kapur SK, Katz AJ (2013) Review of the adipose derived stem cell secretome Biochimie 95: 2222-2228. [PubMed]

34. Bartholomew A, Sturgeon C, Siatskas M, Ferrer K, Mclntosh K, et al. (2002) Mesenchymal stem cells suppress lymphocyte proliferation in vitro and prolong skin graft survival in vivo. Exp Hematol 30: 42-48. [PubMed]

35. Casiraghi F, Azzollini N, Cassis P, Imberti B, Morigi M, et al. (2008) Pretransplant infusion of mesenchymal stem cells prolongs the survival of a semiallogeneic heart transplant through the generation of regulatory $\mathrm{t}$ cells. J Immunol 181 3933-3946. [PubMed]

36. Casiraghi F, Azzollini N, Todeschini $M$, Cavinato RA, Cassis $P$, et al (2012) Localization of mesenchymal stromal cells dictates their immune or proinflammatory effects in kidney transplantation. Am J Transplant 12: 2373 2383. [PubMed]

37. English K, French A, Wood KJ (2010) Mesenchymal stromal cells: Facilitators of successful transplantation? Cell Stem Cell 7: 431-442. [PubMed]

38. Ge W, Jiang J, Baroja ML, Arp J, Zassoko R, et al. (2009) Infusion of mesenchymal stem cells and rapamycin synergize to attenuate alloimmune responses and promote cardiac allograft tolerance. Am J Transplant 9: 1760 1772. [PubMed]

39. Culmes M, Eckstein HH, Burgkart R, Nüssler AK, Guenther M, et al. (2013) Endothelial differentiation of adipose-derived mesenchymal stem cells is improved by epigenetic modifying drug bix-01294. Eur J Cell Biol 92: 70-79. [PubMed] 